BULL. AUSTRAL. MATH. SOC.

VOL. $15(1976), 129-131$.

\title{
A smooth, non-reflexive second conjugate space
}

\section{M.A. Smith}

It is shown that a separable, quasi-reflexive Banach space of deficiency one admits an equivalent norm such that its second conjugate space is smooth; this answers a question raised by Ivan Singer [Bulz. Austral. Math. Soc. 12 (1975), 407-416].

A Banach space $B$ with conjugate space $B^{*}$ is said to be smooth if for every $\approx \in S(B) \equiv\{x \in B:\|x\|=1\}$, there exists a unique $x^{*} \in S\left(B^{*}\right)$ such that $x^{*}(x)=1$.

The purpose of this note is to ohow the existence of a non-reflexive Banach space with smooth second conjugate space, a matter of interest raised by Rainwater [4]. Specifically, we show that the quasi-reflexive space $J$ of James [3] admits an equivalent norm for which the second conjugate space is smooth; this answers a question raised by singer [5].

A Banach space $B$ is said to be very smooth (Sullivan [6]) if for every $x \in S(B)$ there exists a unique $x^{* * *} \in S\left(B^{* * *}\right)$ such that $x^{* * *}(Q(x))=1$ where $Q: B \rightarrow B^{* *}$ is the canonical embedding.

A Banach space $B$ is said to be extremely smooth (Sullivan [6]) if whenever $x^{* *}\left(x^{* *}\right)=y^{* *}\left(x^{* *}\right)=1$ where $x^{* *} \in S\left(B^{* *}\right)$ and $x^{* * *}, y^{* * *} \in S\left(B^{* * *}\right)$ then $x^{* * *}-y^{* * *} \in Q(B)^{*}$.

THEOREM. If $B$ is a separable, quasi-reflexive Banach space of deficiency one, then $B$ admits an equivalent norm such that $B^{* *}$ is smooth.

Received 13 April 1976. 
Proof. Since both $B$ and $B^{*}$ are separable, $B$ admits an equivalent norm that is both Fréchet and uniformly Gâteaux differentiable (see Day [2]). Thus we can and do assume that $B$ has such a norm. It follows from results of Sullivan [6] that $B$ is both very smooth and extremely smooth, since $B$ is Fréchet and uniformly Gâteaux differentiable respectively. Write $B^{* *}=Q(B) \oplus\left[b^{* *}\right]$ where $\left[b^{* *}\right]$ denotes the linear span of $\left\{b^{* *}\right\}$.

We claim that $B^{* *}$ is smooth. To obtain a contradiction, suppose there exist $x^{* *} \in S\left(B^{* *}\right)$ and $x^{* * *}, y^{* * *} \in S\left(B^{* * *}\right)$ such that $x^{* * *} \neq y^{* * *}$ and $x^{* * *}\left(x^{* *}\right)=y^{* *}\left(x^{* *}\right)=1$. Write $x^{* *}=Q(b)+B b^{* *}$ where $b \in B$ and $\beta$ is a scalar. Since $B$ is very smooth, we have that $\beta \neq 0$. Also since $B$ is extremely smooth, we have that $x^{* * *}-y^{* * *} \in Q(B)^{\perp}$. It follows that

$$
0=\left(x^{* * *}-y^{* * *}\right)\left(x^{* *}\right)=\left(x^{* *}-y^{* * *}\right)\left(\beta b^{* *}\right) \text {. }
$$

Since $\beta \neq 0$, it foliows that $\left(x^{* * *}-y^{* * *}\right)\left(b^{* *}\right)=0$, and hence $x^{* * *}=y^{* * *}$. This contradiction completes the proof.

REMARK. From the proof of the theorem, a quasi-reflexive Banach space of deficiency one that is both very smooth and extremely smooth has smooth second conjugate space. However, this implication is not true in general since $c_{0}$ admits an equivalent norm that is both Fréchet and uniformly Gâteaux differentiable, and Day [1] has shown that $\tau^{\infty}$ admits no equivalent smooth norm. It is trivial that a Banach space is very smooth and extremely smooth if its second conjugate space is smooth.

\section{References}

[1] Mahlon M. Day, "Strict convexity and smoothness of normed spaces", Trans. Amer. Math. Soc. 78 (1955), 516-528.

[2] Mahlon M. Day, Normed Zinear spaces, 3rd ed. (Ergebnisse der Mathematik und ihrer Grenzgebiete, 21. Springer-Verlag, Berlin, Heidelberg, New York, 1973).

[3] Robert C. James, "A non-reflexive Banach space isometric with its second conjugate space", Proc. Nat. Acad. Sci. USA 37 (1951), 174-177. 
[4] John Rainwater, "A non-reflexive Banach space has non-smooth third conjugate space", preprint.

[5] I van Singer, "On the problem of non-smoothness of non-reflexive second conjugate spaces", Bull. Austral. Math. Soc. 12 (1975), 407-416.

[6] Francis Sullivan, Geometrical properties determined by the higher duals of a Banach space", preprint.

Department of Mathematics,

Lake Forest Coll lege,

Lake Forest,

III inois, USA. 